\title{
Ground-based photometry of the contact binary V1128 Tauri
}

\author{
G. Taş, S. Evren, Ö. Çakirli, and C. Íbanoğlu
}

Ege University Observatory, 35100 Bornova-Izmir, Turkey

e-mail: [sevren, cakirli,ibanoglu]@astronomy.sci.ege.edu.tr

Received 21 January 2003 / Accepted 22 May 2003

\begin{abstract}
V1128 Tau is a short period W UMa type eclipsing binary which has a visual companion with a separation of $14^{\prime \prime}$ and a difference in brightness of about 1 mag. We observed the system in $B$ and $V$ filters during two observing seasons using three different telescopes and detectors. We obtained a total of 6063 observational points in each colour. The light curve reveals that V1128 Tau has a totality in the primary eclipse, which lasts about $16 \mathrm{~min}$. The shape of the light curve indicates that V1128 Tauri is a W-type W UMa binary. We subtracted the visual component's light contribution to the total light of the system. We used the latest version of the Wilson-Devinney code for the analysis of the light curves and determined for the first time the geometric and physical parameters of the system. We found that the system consists of late $\mathrm{G}$ and early $\mathrm{K}$ type components. The more massive, larger component is cooler by about $300 \mathrm{~K}$ than its companion. The system has a circular orbit with an inclination of $85^{\circ}$. The light curves show a typical O'Connell effect, maximum I being brighter than maximum II. This difference may arise from a cool or hot region on either component and/or an accretion process between the components. Since the components appear to be later than G2, we assumed a cool spot or group of spots on the cooler component. Therefore, the light curves were also analyzed using the spot hypothesis and the results were compared with those obtained with the no-spot model.
\end{abstract}

Key words. stars: activity - stars: individual: V1128 Tau - binaries: eclipsing

\section{Introduction}

V1128 Tau $\left(\mathrm{BD}+12^{\circ}\right.$ 511) was classified as a Beta Lyr type eclipsing binary; its maximum and minimum brightnesses in $V$ passband and spectral type are given in the Hipparcos catalogue as $9.649,10.241$ and G0, respectively. In the SIMBAD database, the magnitudes and spectral type of the system at the maximum were given as $B=10.29, V=9.69$ and G0, respectively. Moreover, Hipparcos gives the light elements of the system as

HJD $($ MinI $)=2448500.0620+0.3053732 \times E$.

Magazzù et al. (1997) noted that V1128 Tau is the optical counterpart of the ROSAT source RXJ 0349.4+1255. V1128 Tau has a visual companion at a distance of $14^{\prime \prime}$ : BD $+12^{\circ} 511 \mathrm{~B}$. Magazzù et al. (1997) proposed that the visual companion is a tenth magnitude G7 star. It is listed as V1128 Tau in the special list of variable stars (Kazarovets et al. 1999) and classified as a W UMa type eclipsing binary.

In this paper, we present the first ground-based photometric observations of V1128 Tau. In Sect. 2 we give details of the observations and derived light elements. In Sect. 3 we describe the subtraction of the light of the visual companion from the total light of the system. We present the results of the light curve

Send offprint requests to: $\mathrm{G}$. Taş,

e-mail: tas@astronomy.sci.ege.edu.tr analysis with the Wilson-Devinney code in Sect. 4. In the last section, we discuss the nature of the system on the basis of the new results.

\section{Observations}

We observed V1128 Tau during two observing seasons in 2001 and 2002. In the first observing season we made observations of the system between November 22, 2001 and February 9, 2002 using three different detectors and telescopes. We obtained the light curves, which have full phase coverage, in two nights. We continued the observations to obtain the timings of the primary and the secondary minima. The observations were carried out mainly with the $48 \mathrm{~cm}$ Cassegrain telescope equipped with a high-speed three-channel photometer (HSTCP), using three cooled Hamamatsu R1463P PMTs. Because of the optical design of HSTCP we could use only one comparison star. Therefore, some observations were made with the $30 \mathrm{~cm}$ Schmidt-Cassegrain telescope equipped with a SSP-5 photometer (including Hamamatsu R4457 PMT) at Ege University Observatory (EUO) to check whether the light of the comparison star changes or not. The system has also been observed with the $150 \mathrm{~cm}$ Cassegrain telescope using a CCD camera at TUBITAK National Observatory (TUG). We obtained 3618 observing points in each of the $B$ and $V$ filters at EUO, and 1784 points in the $V$ filter at TUG. The second 
Table 1. The photometric observations.

\begin{tabular}{cccc}
\hline \hline $\begin{array}{c}\text { JD range } \\
(2400000+)\end{array}$ & $\begin{array}{c}\text { Obs. } \\
\text { nights }\end{array}$ & $\begin{array}{c}\text { Obs. } \\
\text { points }\end{array}$ & $\begin{array}{c}\text { Observatory } \\
\text { and detector }\end{array}$ \\
\hline 52236 & Nov. 22, 2001 & 266 & EUO / SSP-5 \\
52240 & Nov. 26, 2001 & 184 & EUO / SSP-5 \\
52248 & Dec. 4, 2001 & 368 & EUO / HSTCP \\
52254 & Dec. 10, 2001 & 283 & EUO / HSTCP \\
52258 & Dec. 14, 2001 & 933 & EUO / HSTCP \\
52263 & Dec. 19, 2001 & 986 & EUO / HSTCP \\
52277 & Jan. 2, 2002 & 413 & EUO / HSTCP \\
52277 & Jan. 2, 2002 & 185 & EUO / SSP-5 \\
52313 & Feb. 7, 2002 & 744 & TUG / CCD \\
52314 & Feb. 8, 2002 & 639 & TUG / CCD \\
52315 & Feb. 9, 2002 & 401 & TUG / CCD \\
52536 & Sep. 18, 2002 & 214 & EUO / HSTCP \\
52563 & Oct. 15, 2002 & 406 & EUO / HSTCP \\
52565 & Oct. 17, 2002 & 307 & EUO / HSTCP \\
52608 & Nov. 29, 2002 & 274 & EUO / HSTCP \\
\hline
\end{tabular}

observing season covers the time interval between September 18 and November 29, 2002. The $48 \mathrm{~cm}$ telescope was used in this season. A total of 1201 observing points were obtained in each colour during the second season. Details of the observations are summarized in Table 1.

We used BD $+12^{\circ} 513\left(B=10^{\mathrm{m}} \cdot 49, V=8 \mathrm{~m} \cdot 93\right.$, Sp. K5) and $\mathrm{BD}+12^{\circ} 512\left(B=11^{\mathrm{m}} 3, V=9 \mathrm{~m} .84, \mathrm{Sp} . \mathrm{K} 0\right)$ as the comparison and the check stars, respectively. We observed both the comparison and the check star with the $30 \mathrm{~cm}$ telescope. These observations indicated that the light of the comparison star was constant. The counts from the variable, the comparison star and the sky background were simultaneously recorded using an integration time of ten seconds. Following traditional reduction procedure, we obtained differential magnitudes, in the sense variable minus comparison, and then corrected for atmospheric extinction. The extinction coefficients were calculated for each band using the observed magnitudes of the comparison star. The times were also reduced to the Sun's center. The standard deviation of each observed point is approximately 0.010 in $B$ and 0 . 008 in $V$ and is almost completely determined by Poisson statistics of stored counts.

The HSTCP seems to be suitable for time-series multicolour photometry of rapid variables. All three channels of the photometer are aligned with the telescope's main axis. The rotational bearing which is one of the main units of the HSTCP was designed to rotate the whole photometer around the main axis of the telescope (target star). It enables one to search out a comparison star, in combination with the radial movement of the second channel. The movable field eyepiece unit makes it possible to survey the field around the target star as well as to search the comparison star by moving the field eyepiece in the radial direction. The second-channel moving unit enables us to intercept the light of a comparison star into the aperture of this channel according to the field eyepiece position when this star

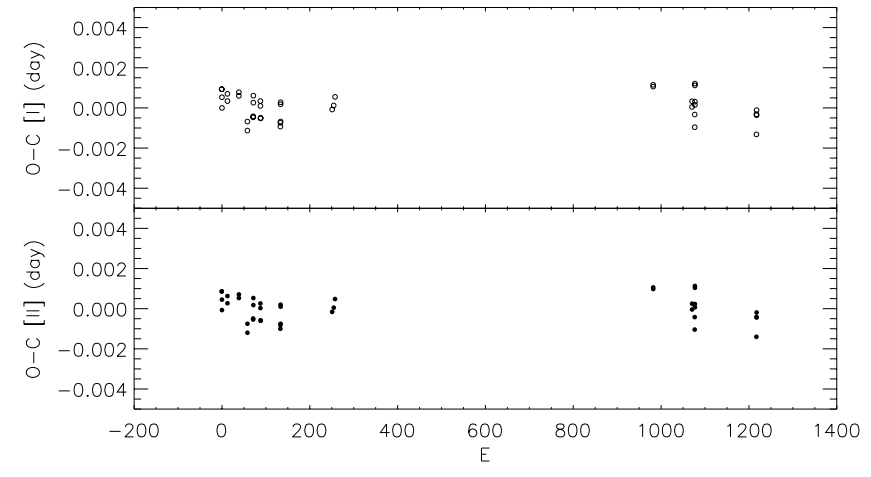

Fig. 1. The O-C variation of V1128 Tauri calculated from Eq. (1) (top panel) and Eq. (2) (bottom panel).

is found. The third-channel movement unit is designed for the cases when the configuration of all three channels does not allow to get a pure sky background in the third channel, i.e. when an interfering star is seen in the third channel.

This unit makes it possible to move the third channel to the new position without changing the position of the first two channels. Since HSTCP is a three-channel photometer with three-channel it yields synchronous observations of the variable, the comparison star and the sky background and it allows fast photometry. We were therefore able to obtain many data points on the one night that allowed high sensitivity. The details on HSTCP can be found in Kalytis (1999).

All measurements of the variable obtained at EUO include light of the faint visual companion. We also made time-series CCD observations of V1128 Tau with the $150 \mathrm{~cm}$ telescope, which could easily separate the visual companion. Therefore, the visual companion was used as comparison star. The reduction was made using the IRAF $^{1} /$ CCDRED package.

During the two observing seasons 19 times of mid-primary minimum and 21 times of mid-secondary minimum were obtained; they are given in Table 2. The times of mid-eclipses were derived by the method of Kwee \& van Woerden (1956). Since the ingress and egress of the primary and secondary minima are nearly symmetric and deep enough, standard deviations of mid-minimum times are rather small, given in parenthesis in the first column, except for the JD 2452608 observing night because of bad weather conditions. A linear ephemeris (Eq. (1)) was applied to obtain differences between observed and computed times.

To correct the light elements, we applied the linear least squares method to $\mathrm{O}-\mathrm{C}(\mathrm{I})$ values of the primary and secondary minima using equal weights for each. The new light elements we determined are

HJD $($ MinI $)=2452236.60963( \pm 14)+0.3053732( \pm 2) \times E .(2)$

We show the $\mathrm{O}-\mathrm{C}$ (I) diagram obtained using Eq. (1) for V1128 Tau at the top panel in Fig. 1. The computed residuals using light elements in Eq. (2) are shown in the bottom panel

\footnotetext{
${ }^{1}$ IRAF is distributed by National Optical Astronomy Observatory, which is operated by the Association of University for Research in Astronomy, inc. (AURA) under cooperative agreement with the NSF (National Science Foundation).
} 
Table 2. The times of light minimum for V1128 Tau.

\begin{tabular}{|c|c|c|c|c|c|}
\hline $\begin{array}{l}\text { JD (Hel). } \\
(2400000+)\end{array}$ & $E$ & $\begin{array}{l}\text { O-C (I) } \\
\text { (days) }\end{array}$ & $\begin{array}{c}\text { O-C (II) } \\
\text { (days) }\end{array}$ & Filter & Type \\
\hline $52236.45780( \pm 19)$ & -0.5 & 0.00093 & 0.00086 & $V$ & II \\
\hline $52236.45781( \pm 37)$ & -0.5 & 0.00093 & 0.00086 & $B$ & II \\
\hline $52236.60956( \pm 34)$ & 0.0 & 0.00000 & -0.00007 & $V$ & I \\
\hline $52236.61009( \pm 39)$ & 0.0 & 0.00053 & 0.00045 & $B$ & I \\
\hline $52240.42707( \pm 8)$ & 12.5 & 0.00034 & 0.00027 & $V$ & II \\
\hline $52240.42743( \pm 9)$ & 12.5 & 0.00070 & 0.00063 & $B$ & II \\
\hline $52248.36703( \pm 7)$ & 38.5 & 0.00060 & 0.00053 & $V$ & II \\
\hline $52248.36721( \pm 8)$ & 38.5 & 0.00078 & 0.00071 & $B$ & II \\
\hline $52254.32008( \pm 7)$ & 58.0 & -0.00113 & -0.00120 & $V$ & I \\
\hline $52254.32053( \pm 7)$ & 58.0 & -0.00068 & -0.00075 & $B$ & I \\
\hline $52258.29059( \pm 7)$ & 71.0 & -0.00047 & -0.00054 & $V$ & I \\
\hline $52258.29064( \pm 7)$ & 71.0 & -0.00042 & -0.00049 & $B$ & I \\
\hline $52258.44400( \pm 8)$ & 71.5 & 0.00026 & 0.00018 & $B$ & II \\
\hline $52258.44435( \pm 8)$ & 71.5 & 0.00061 & 0.00053 & $V$ & II \\
\hline $52263.32982( \pm 8)$ & 87.5 & 0.00010 & 0.00003 & $V$ & II \\
\hline $52263.33005( \pm 8)$ & 87.5 & 0.00034 & 0.00026 & $B$ & II \\
\hline $52263.48188( \pm 8)$ & 88.0 & -0.00052 & -0.00060 & $B$ & I \\
\hline $52263.48190( \pm 8)$ & 88.0 & -0.00050 & -0.00058 & $V$ & I \\
\hline $52277.22327( \pm 52)$ & 133.0 & -0.00093 & -0.00100 & $B$ & I \\
\hline $52277.22346( \pm 62)$ & 133.0 & -0.00074 & -0.00081 & $B$ & I \\
\hline $52277.37620( \pm 55)$ & 133.5 & -0.00068 & -0.00075 & $B$ & II \\
\hline $52277.37706( \pm 9)$ & 133.5 & 0.00018 & 0.00010 & $V$ & II \\
\hline $52277.37716( \pm 9)$ & 133.5 & 0.00028 & 0.00020 & $B$ & II \\
\hline $52313.25815( \pm 4)$ & 251.0 & -0.00008 & -0.00016 & $V$ & I \\
\hline $52314.32716( \pm 4)$ & 254.5 & 0.00012 & 0.00005 & $V$ & II \\
\hline $52315.24371( \pm 5)$ & 257.5 & 0.00055 & 0.00048 & $V$ & II \\
\hline $52536.48710( \pm 10)$ & 982.0 & 0.00106 & 0.00098 & $V$ & I \\
\hline $52536.48719( \pm 17)$ & 982.0 & 0.00115 & 0.00106 & $B$ & I \\
\hline $52563.51161( \pm 7)$ & 1070.5 & 0.00005 & -0.00004 & $V$ & II \\
\hline $52563.51190( \pm 9)$ & 1070.5 & 0.00033 & 0.00025 & $B$ & II \\
\hline $52565.34285( \pm 12)$ & 1076.5 & -0.00096 & -0.00104 & $B$ & II \\
\hline $52565.34347( \pm 15)$ & 1076.5 & -0.00033 & -0.00042 & $V$ & II \\
\hline $52565.49665( \pm 7)$ & 1077.0 & 0.00015 & 0.00007 & $V$ & I \\
\hline $52565.49681( \pm 7)$ & 1077.0 & 0.00031 & 0.00023 & $B$ & I \\
\hline $52565.49761( \pm 26)$ & 1077.0 & 0.00112 & 0.00104 & $B$ & I \\
\hline $52565.49770( \pm 59)$ & 1077.0 & 0.00121 & 0.00113 & $V$ & I \\
\hline $52608.24742( \pm 140)$ & 1217.0 & -0.00132 & -0.00140 & $B$ & I \\
\hline $52608.24842( \pm 105)$ & 1217.0 & -0.00032 & -0.00041 & $V$ & I \\
\hline $52608.40107( \pm 86)$ & 1217.5 & -0.00036 & -0.00044 & $V$ & II \\
\hline $52608.40132( \pm 214)$ & 1217.5 & -0.00011 & -0.00019 & $B$ & II \\
\hline
\end{tabular}

of this figure. The orbital period derived by us is found to be equal to that given by Hipparcos.

We used the magnitude and $B-V$ colour of the comparison star, taken from SIMBAD, for transformation to the standard system. The standard magnitudes and $B-V$ colours of

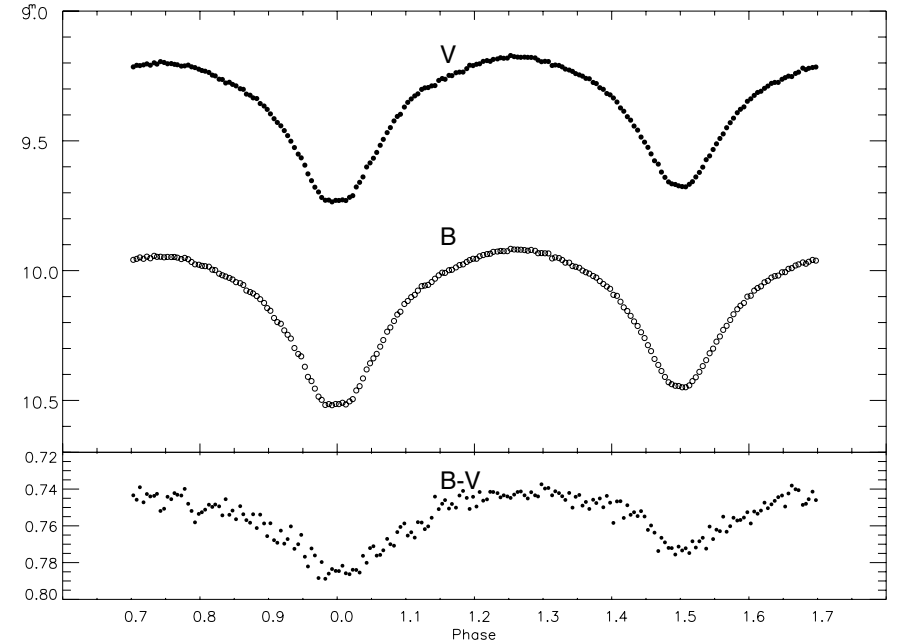

Fig. 2. The light and colour curves of V1128 Tau including the light contribution of the visual companion.

the system were plotted against the orbital phase, computed with the corrected light elements, and are shown in Fig. 2. The observational data is available upon request from the authors. As can be seen clearly from Fig. 2, the primary minimum is a total eclipse. The duration of the totality is about $16 \mathrm{~min}$, corresponding to $0^{\mathrm{p}} .031$.

\section{The visual companion of V1128 Tau $\left(B D+12^{\circ}\right.$ 511B)}

Since the visual companion is too close to be separated by the diaphragm we measured the light of both stars. We used CCD observations to subtract the contribution of the visual companion's light to the total light of the system. In four special phases, namely $0.0,0.25,0.50,0.75$, we were able to measure the magnitude differences between the variable and its visual companion using CCD observations. Since we have the total brightness of the eclipsing pair plus visual companion, we estimated the $V$ magnitude of the visual companion. Thus, the apparent magnitude of the visual companion was estimated to be about 10.856 in $V$. However, its visual magnitude was given by SIMBAD as $10^{\mathrm{m}} 6$. Using $B-V$ for the visual companion taken from SIMBAD database we find its blue magnitude to be $11 \mathrm{~m} 556$. As can be seen from Fig. 2, the maximum brightness of the eclipsing pair plus visual companion is $B(123)=9$ m.921 and $V(123)=9$ m 176 . At the mid-primary minimum (the cooler component is in front of the hotter one), we have a common contribution of cooler and visual components because the hotter component is completely eclipsed: $B(13)=10^{\mathrm{m}} .514$ and $V(13)=9 \mathrm{~m} .730$. If we use the magnitude differences, variable minus visual companion, measured from CCD observation, we easily compute the magnitudes of the components separately and also compute the contribution of each component to the total light.

When we calculate their $B-V$ colours, we can see that the hotter and smaller component of the eclipsing pair and the visual companion have approximately the same spectral type, and the more massive primary component is cooler than both 
Table 3. The blue and visual magnitudes of the components and their contributions to the total light.

\begin{tabular}{cccccc}
\hline \hline Star & $B(\mathrm{mag})$ & $L / L_{\text {tot }}(B)$ & $V(\mathrm{mag})$ & $L / L_{\text {tot }}(V)$ & $B-V(\mathrm{mag})$ \\
\hline Hotter & 10.859 & 0.421 & 10.154 & 0.403 & 0.705 \\
Cooler & 11.039 & 0.357 & 10.205 & 0.385 & 0.834 \\
Visual component & 11.556 & 0.222 & 10.856 & 0.211 & 0.700 \\
\hline
\end{tabular}

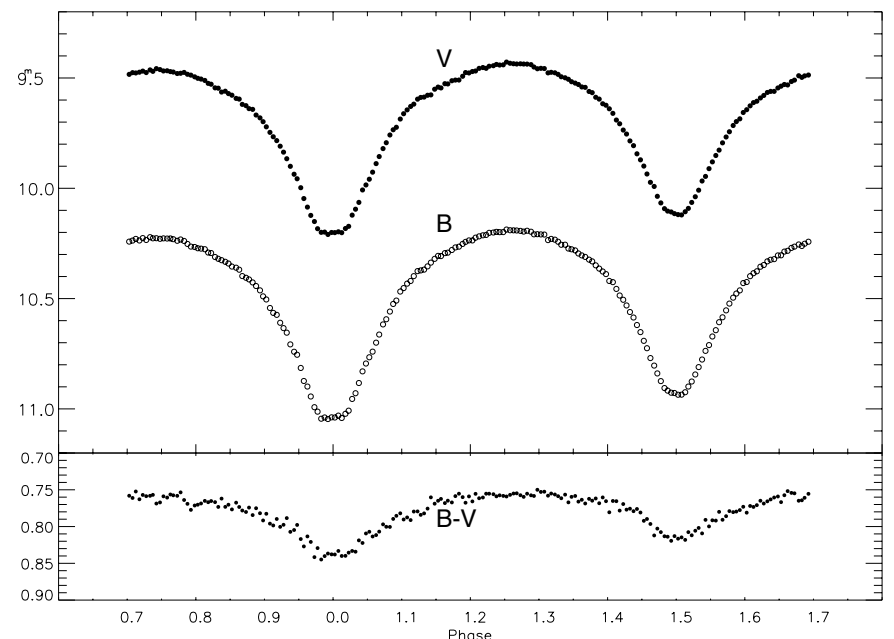

Fig. 3. The light and colour curves of V1128 Tauri, after subtraction of the light contribution of the visual companion.

of them. $B, V$ magnitudes and $B-V$ colours for the components of the eclipsing pair and the visual companion are given in Table 3, respectively. We also give the light contribution of each component to the the total light of the system in this table.

\section{The analysis of the light curves}

When we subtract the contribution of the visual companion to the total light of the system the depth of primary eclipse changes from 0.593 to 0.851 and from 0.553 to 0.772 , in $B$ and $V$, respectively. In Fig. 3, we present the light curves in $B$ and $V$, and the $B-V$ colour curve after removing the contribution of the visual companion's light. The continuous light change at out-of-eclipse portions of the light curves indicates strong gravitational distortions, as expected in contact binaries. Our light curves show that V1128 Tauri is a W UMa type, since these show strong curved maxima. The colour curves show reddening in both minima, and the depths of the minima are approximately the same, which is typical for a W UMa type.

We did not notice any difference between the light curves obtained in two successive observing seasons. Therefore, we averaged all observed points within a phase interval of 0.05 and normalized to the light maximum, namely with the magnitude at phase 0.25 , for each filter. $B$ and $V$ light curves consisting of normal points were solved separately and simultaneously by using the latest version of the Wilson-Devinney code, first presented in 1971 by Wilson \& Devinney (1971) and revised by Wilson (1994).

For a contact binary, the mass ratio $q=m_{2} / m_{1}$ can be inferred from the light curve analysis, which also determines the shape of the light curve. There is no previous light curve solution for this system. Therefore, we tried to estimate preliminary elements by comparing the light curves with those of contact systems. From these initial hints and by trial and error, we constructed a set of parameters able to simulate the observed curves. Since the DC procedure converges faster when the mass ratio is less than 1 , we introduced a phase shift of 0.5 . The differential correction code was run until the output corrections were smaller than the probable errors $\sigma$ of the elements. Since there is no spectroscopic study of the system we started to solve the light curve using different values for the mass ratio of the system. We obtained light curve solutions for specific values of the mass ratio in a range from 0.3 to 0.9 . The sum of squares of residuals reached a minimum for $q=0.48$. Mochnacki \& Doughty (1972a) developed a technique for obtaining models for totally-eclipsing W UMa systems. We estimated the internal contact angle to be $6^{\circ} \pm 1^{\circ}$ directly from the observations. Using this value and the orbital inclination we estimated the mass ratio to be about 0.47 , following the technique proposed by Mochnacki \& Doughty (1972a,b). The final check of this value for the mass ratio can only be made from spectroscopic observations. The fill-out ratio was set at 1.0. Therefore, we adopted $q=0.48$ and kept it fixed throughout the analysis. The other adopted parameters in the solution are the following: we assumed $T_{1}=5236 \mathrm{~K}$ for star 1 (the more massive primary, eclipsed during the secondary minimum), which corresponds to a $B-V$ colour of 0.834 (Gray 1992), limb darkening coefficients $x_{1}=x_{2}=0.839$ and 0.744 for $B$ and $V$ band, respectively (Díaz-Cordovés et al. 1995), gravity darkening coefficients $g_{1}=g_{2}=0.32$ (Lucy 1967) and albedo values $A_{1}=A_{2}=0.5$ (Rucinski 1969). The subscripts refer to the components of the binary. The adjustable parameters are the orbital inclination, $i$, the effective temperature of star $2, T_{2}$, the potential of the components, $\Omega_{1}$ and $\Omega_{2}$, and the monochromatic luminosity of star $1, L_{1}$ (the Planck function is used to compute the luminosity). Assuming it is a contact binary we used Mode 3 of the DC program.

The computed light curves are compared with the observations in Fig. 4. As we can see from these figures the maxima are not equal in height. The unequal light level at the quadratures, (the O'Connell effect), is known in many eclipsing binaries and several suggestions have been made to explain this effect by different authors. For V1128 Tauri, maximum II is fainter than maximum I. The brightness difference at the quadratures, in the sense of maximum II minus I, is significant and amounts to $0^{\mathrm{m}} 042$ in the $B$, and $0^{\mathrm{m}} 040$ in the $V$ band. This difference in height of the maxima may be due to surface inhomogenities of the components, such as a cool or hot region on either component and/or the accretion process between the components, as suggested recently by Rucinski (1997). In most solar-like 
Table 4. The results of the photometric solutions.

\begin{tabular}{ccccccc}
\hline \hline Parameters & $B$ (unspotted) & $V$ (unspotted) & $B$ (spotted) & $V$ (spotted) & $B V$ (unspotted) & $B V$ (spotted) \\
\hline$q\left(m_{2} / m_{1}\right)$ & 0.48 & 0.48 & $0.49(1)$ & $0.48(1)$ & 0.48 & $0.48(1)$ \\
$i$ & $86.65(42)$ & $84.16(35)$ & $86.81(28)$ & $84.15(24)$ & $85.32(17)$ & $85.46(13)$ \\
$T_{1}(\mathrm{~K})$ & 5236 & 5236 & 5236 & 5236 & 5236 & 5236 \\
$T_{2}(\mathrm{~K})$ & $5537(7)$ & $5515(8)$ & $5510(6)$ & $5515(6)$ & $5531(6)$ & $5528(4)$ \\
$\Omega$ & $2.814(4)$ & $2.818(3)$ & $2.802(1)$ & $2.816(2)$ & $2.817(3)$ & $2.825(2)$ \\
$L_{1}$ & $6.788(23)$ & $7.078(23)$ & $6.872(19)$ & $7.126(16)$ & $6.772(18)(B)$ & $6.836(14)(B)$ \\
& & & & & $7.078(15)(V)$ & $7.129(12)(V)$ \\
$L_{2}$ & 4.839 & 4.642 & 4.936 & 4.679 & $4.801(B)$ & $4.883(B)$ \\
& & & & & $4.702(V)$ & $4.775(V)$ \\
$x_{1}=x_{2}$ & 0.839 & 0.744 & 0.839 & 0.744 & $0.839(B)$ & $0.839(B)$ \\
& & & & $0.744(V)$ & $0.744(V)$ \\
$A_{1}=A_{2}$ & 0.5 & 0.5 & 0.5 & 0.5 & 0.5 & 0.5 \\
$g_{1}=g_{2}$ & 0.32 & 0.32 & 0.32 & 0.32 & 0.32 & 0.32 \\
$r_{1}$ (pole) & $0.4217(6)$ & $0.4209(5)$ & $0.4251(2)$ & $0.4217(5)$ & $0.4212(5)$ & $0.4209(4)$ \\
$r_{1}$ (side) & $0.4492(8)$ & $0.4481(7)$ & $0.4537(3)$ & $0.4491(7)$ & $0.4485(6)$ & $0.4482(16)$ \\
$r_{1}$ (back) & $0.4786(11)$ & $0.4771(9)$ & $0.4852(5)$ & $0.4787(10)$ & $0.4776(8)$ & $0.4778(8)$ \\
$r_{2}$ (pole) & $0.3008(7)$ & $0.2999(6)$ & $0.3070(4)$ & $0.3014(7)$ & $0.3002(5)$ & $0.3022(5)$ \\
$r_{2}$ (side) & $0.3145(8)$ & $0.3134(6)$ & $0.3217(5)$ & $0.3152(8)$ & $0.3138(6)$ & $0.3160(6)$ \\
$r_{2}$ (back) & $0.3498(12)$ & $0.3481(10)$ & $0.3606(9)$ & $0.3507(14)$ & $0.3487(9)$ & $0.3515(10)$ \\
$\Sigma$ & 0.105 & 0.067 & 0.054 & 0.033 & 0.194 & 0.111 \\
$\phi$ (deg) & & & 90 & 90 & & 90 \\
$\theta$ (deg) & & & 266 & 266 & & 48 \\
$r_{\text {spot }}$ (deg) & & & 48 & 48 & & 0.986 \\
$T_{\text {spot }} / T_{*}$ & & & 0.986 & 0.985 & &
\end{tabular}

stars the cause of brightness variation was attributed to cool spots. Since the spectral types of the components appear to be later than G2, we assumed cool spots on either component as the cause of asymmetry in the the light curve, e.g., unequal maxima. Finally, we solved the light curves assuming spot or spot groups located onthe cooler component. The parameters found for the unspotted and spotted solutions are given in Table 4. This analysis indicates that a spot on the cooler component at $266^{\circ}$ longitude covers almost one-fourth of this component. The computed curves of both solutions were compared with the observations in Figs. 4 and 5. These figures show that there is a good agreement between computed and observed curve, except at both side of the second maximum.

\section{Results and discussion}

Although a large number of studies has been published on contact binaries, a lot of problems concerning their structure and evolution are still waiting for solution. V1128 Tauri has a visual component, which is more distant than the eclipsing pair according to the data given by SIMBAD. The brightness of V1128 Tau was measured with and without this optical companion. We estimated the light contribution of the visual component to the total light as $29 \%$ in $B$ and $27 \%$ in $V$ band. Subtracting the light contribution of the visual companion we obtained the light variation of the eclipsing pair. Analysing this light variation, on the assumption of a large cool spot region on the cooler component of the binary, the orbital parameters

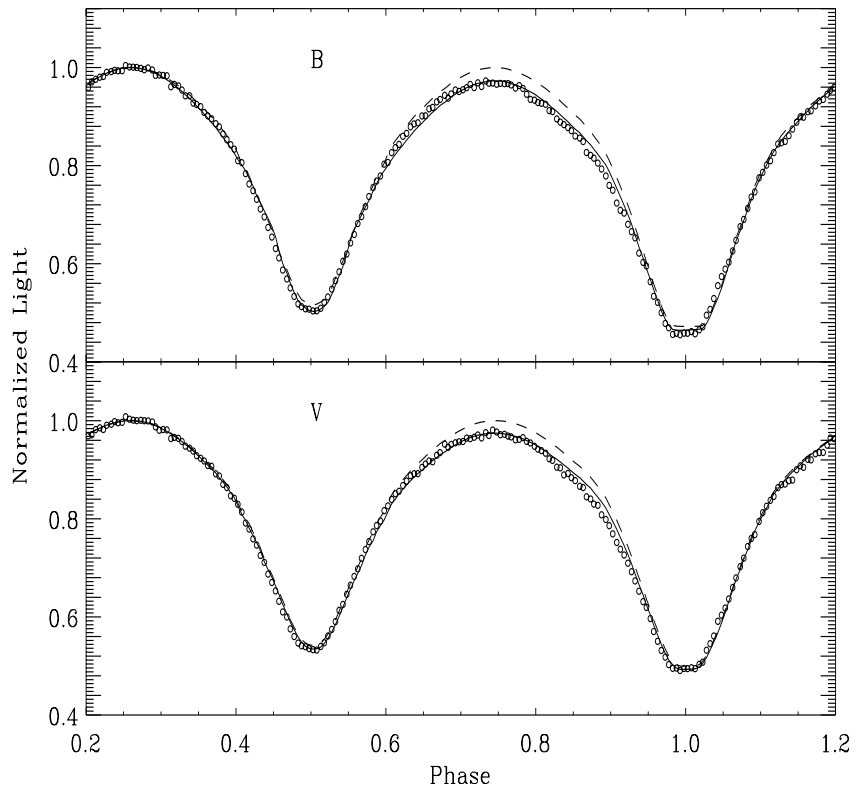

Fig. 4. Unspotted (dashed line) and spotted (continuous line) solutions of V1128 Tau for $B$ and $V$ light curves obtained using the Wilson-Devinney code. The open circles show normal points.

of the system were obtained. We should also state that similar results may be obtained with a hot spot solution by displacing the spot by $180^{\circ}$ in longitude on the same component. The system consists of late $G$ and early K-type stars with a mass ratio of 0.48 , and a temperature difference of about 
$300 \mathrm{~K}$. The fill-out factor of $9 \%$ is somewhat low for W UMa W-type binaries, which usually fall in the 10\% to $20 \%$ range. On the other hand, the system must have been in physical contact long enough to develop good thermal contact as indicated by the small temperature difference. The cooler component has a larger radius than the hotter one and contributes $58 \%$ in $B$ and $60 \%$ in $V$ to the total light. Rucinski (1985) pointed out that the mean mass ratio for $\mathrm{W}$ - subtype systems is greater than that of A - subtype systems. The effective temperature of a secondary in a $\mathrm{W}$ - subtype is higher than that of primary. This is called the $\mathrm{W}$ - subtype phenomenon. For $\mathrm{W}$ - subtypes the radii of the secondaries are 1.5 times larger than for mainsequence systems of the same mass. The primary components of the shallow contact $\mathrm{W}$ - type W UMa systems are unevolved main sequence stars, while those of the deeper contact $\mathrm{A}$ - type systems are near to the terminal-age main sequence (Hilditch et al. 1988). Most theories of the evolution of contact binaries predict that the mass ratio decreases with increasing contact age. The average mass ratio determined for the system is in support of the notion that it has recently come into contact due to angular momentum loss of this spot-active binary.

The observed asymmetry in the light curve has been attributed to the cool spot on the cooler component of the binary. The spot located on the equator is best viewed by the observer at orbital phase 0.70 . However, the light curve can also be reproduced by a hot spot region on the same star displaced by $180^{\circ}$ in longitude. Hot spots on contact systems are generally explained by a gas stream between the components (Rucinski 1997) and/or by white light faculae rather than by dark spots (Guinan 1990). This puzzle still remains unsolved, not only for V1128 Tau, but for many W UMa type stars.
Acknowledgements. The authors are indebted to Dr. S. Rucinski for useful hints which led to a final improvement of the paper. They also thank the director and staff of the TUBITAK National Observatory, who provided us with observing time with the $1.5 \mathrm{~m}$ telescope. This study has made use of the SIMBAD database, operated at CDS, Strasbourg, France. Ö. Çakirli is grateful to KOÇ VAKFI for his educational-background supporting.

\section{References}

Díaz-Cordovés, J., Claret, A., \& Giménez, A. 1995, A\&AS, 110, 329 Gray, D. F. 1992, The observation and analysis of stellar photospheres (Cambridge Astrophysics Series), 431

Guinan, E. F. 1990, in Proc. International Symp. on Evolution in Astrophys., ESA SP-310, 73

Hilditch, R. W., King, D. J., \& McFarlane, T. M. 1988, MNRAS, 231, 341

Kalytis, R. 1999, Tr. J. Phys., 23, 347

Kazarovets, A. V., Samus, N. N., Durlevich, O. V., et al. 1999, IBVS, 4659

Kwee, K. K., \& van Woerden, H. 1956, BAN, 12, 327

Lucy, L. B. 1967, Zert. Astrophys., 65, 89

Magazzù, A., Martin, E. L., Sterzik, M. F., et al. 1997, A\&AS, 124, 449

Mochnacki, S. W., \& Doughty, N. A. 1972a, MNRAS, 156, 51

Mochnacki, S. W., Doughty, N. A. 1972b, MNRAS, 156, 243

Rucinski, S. M. 1969, A\&A, 19, 245

Rucinski, S. M. 1985, Interacting Binary Stars, ed. J. E. Pringle, \& R. A. Wade (Cambridge University Press), 85

Rucinski, S. M. 1997, AJ, 113, 1112

Wilson, R. E., \& Devinney, E. J. 1971, ApJ, 166, 605

Wilson, R. E. 1994, PASP, 106, 921 